\title{
Correlation between time from injury to surgery and the prevalence of ramp and hidden lesions during anterior cruciate ligament reconstruction. A new diagnostic algorithm
}

\author{
Gianni Di Vico' \\ Sigismondo Luca Di Donato ${ }^{2}$ \\ Giovanni Balato² \\ Gaetano Correra² \\ Alessio D'Addona ${ }^{2}$ \\ Nicola Maffulli3 \\ Donato Rosa ${ }^{2}$ \\ 1 Orthopaedic Unit, S. Michele Clinic, Caserta, Italy \\ 2 Orthopaedic Unit, Department of Public Health, \\ School of Medicine, University of Naples Federico II, \\ Naples, Italy \\ 3 Department of Musculoskeletal Disorders, Faculty of \\ Medicine and Surgery, University of Salerno, Salerno, \\ Italy; Centre for Sports and Exercise Medicine, Mile \\ End Hospital, Barts and The London School of \\ Medicine and Dentistry, London, UK
}

Corresponding Author:

Sigismondo Luca Di Donato

Department of Public Health,Orthopaedic Unit,

School of Medicine, "Federico II" University of Naples

Via Sergio Pansini 5, Building 12

80131 Naples, Italy

E-mail: sigismondodidonato@gmail.com

\section{Summary}

Background: Anterior cruciate ligament tears $(A C L)$ are associated with meniscal lesions, that could involve the posterior horn of the medial meniscus (PHMM). A variety of techniques has been proposed to better visualise the postero-medial (PM) compartment. The aim of the study is to evaluate the prevalence of longitudinal tears of peripheral attachment of the PHMM during arthroscopic ACL reconstruction, and to describe a diagnostic algorithm.

Methods: 115 patients who underwent arthroscopic ACL reconstruction were enrolled for the study. An anterior and an intercondylar notch visualisation were ordinarily performed. A postero-medial (PM) portal was performed when the instability of the posterior horn was detected. Statistical significance was assessed by a Chi-squared or Fisher's Exact Test for categorical variables, and by a MannWhitney $\mathrm{U}$ test for continuous variables.
Results: We recorded a $9.6 \%$ prevalence of lesions of the peripheral attachment of PHMM. Nine ramp lesions and two hidden lesions were diagnosed. Patients treated within 6 months from injury, revealed a statistically significant correlation with a higher prevalence of these lesions.

Conclusion: Ramp and hidden lesions are very common ACL rupture associated injuries. Our diagnostic algorithm is a valid and safe option to diagnose these kinds of lesions. A correlation between a longer time from injury than 6 months and a reduced prevalence of these lesions was recorded in our population.

Level of evidence: IV.

KEY WORDS: ACL reconstruction, arthroscopy, hidden lesion, knee, meniscus, ramp lesion.

\section{Background}

Anterior cruciate ligament tears (ACL) are often associated with meniscal lesions ${ }^{1,2}$, that could involve the posterior horn of the medial meniscus (PHMM) ${ }^{3,4}$. The term "ramp lesion" was first used by Strobel ${ }^{5}$ to identify a characteristic longitudinal tear of meniscosynovial and meniscocapsular junction of PHMM in patients who underwent ACL arthroscopic reconstruction, with a prevalence between 9 and $17 \% \%^{6,7}$. The term "hidden" lesion, instead, defines another characteristic lesion of the peripheral attachment of PHMM, that can be diagnosed only after a soft tissue debridement ${ }^{8}$ (Fig. 1 a, b). A variety of techniques, such as intercondylar notch visualisation through anterior arthroscopic portals ${ }^{9}$, or the use of a postero-medial (PM) portal ${ }^{8,10}$, have been proposed to better visualise the PM compartment.

The aim of this prospective study is to evaluate the prevalence of lesions of the peripheral attachment of the PHMM in a consecutive series of patients who underwent arthroscopic ACL reconstruction in correlation with the time between injury and surgery. Furthermore a diagnostic algorithm was described, which considers the PM compartment systematic exploration through intercondylar notch visualisation, and the use of a PM portal and soft-tissue debridement only in the presence of PHMM instability. We hypothesised that our 3-step algorithm improves the diagnosis of ramp and hidden lesions of the PHMM compared with a standard anterior visualization. 


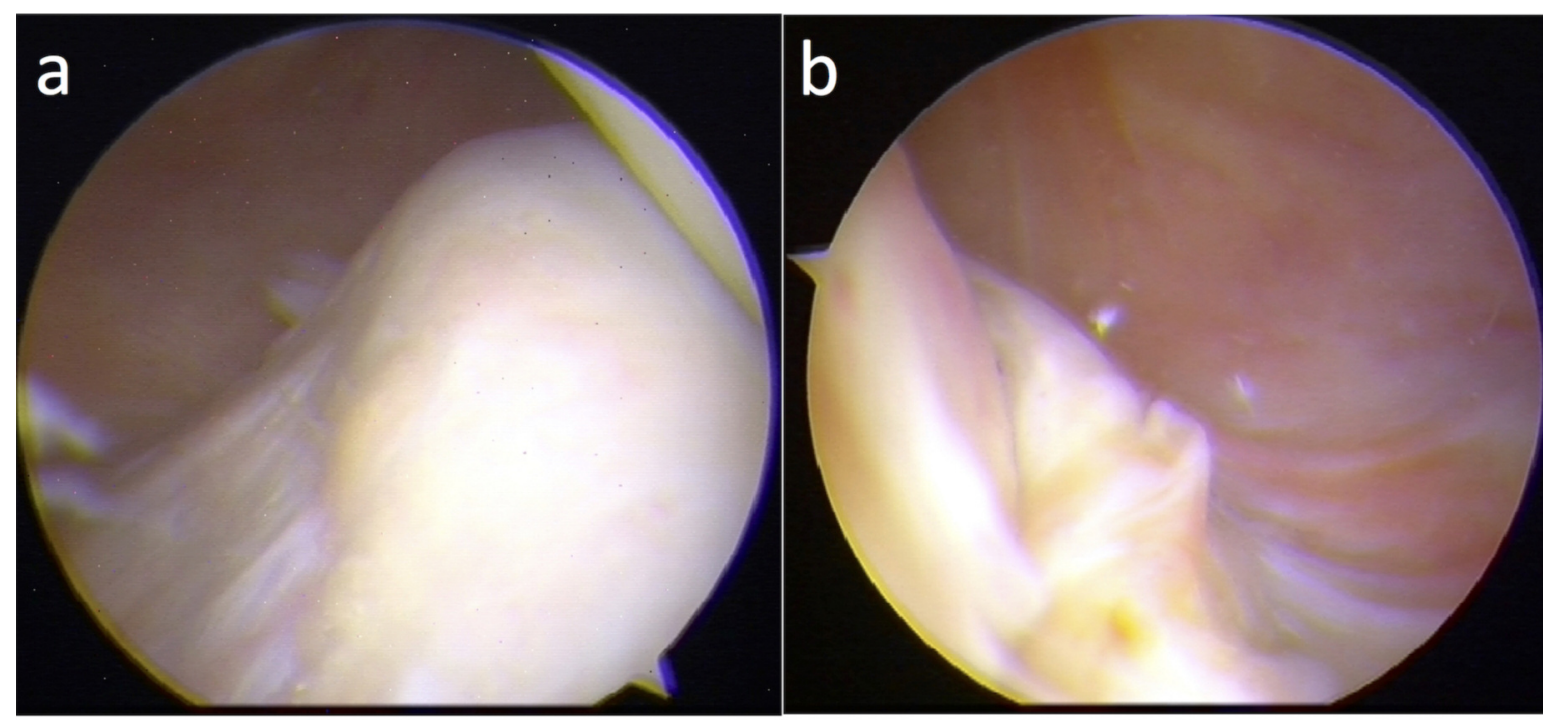

Figure $1 \mathrm{a}$, b. Intraoperative images. A healthy posterior horn of medial meniscus peripheral attachment (a); a ramp lesion (b).

\section{Materials and methods}

We examinated a total of 139 patients for this study who underwent arthroscopic ACL reconstruction from January 2015 to March 2016. A systematic evaluation of the PM compartment was performed during arthroscopic procedure.

The inclusion criterion was: patients with clinical and instrumental signs (X-plain radiography and Magnetic Resonance Imaging) of traumatic acute and chronic $A C L$ tear who underwent arthroscopic ACL reconstruction.

The exclusion criteria were: ACL revision surgery; history of previous knee surgery; patients who underwent other procedures during ACL reconstruction, such as other ligament reconstruction or high tibial osteotomy; knee dislocations.

115 patients were enrolled for this study according to our inclusion criteria.

24 patients were excluded because 10 patients underwent revision surgery, 6 had history of previous knee surgery, 7 underwent other procedures, 1 reported a knee dislocation.

Data about age, gender, and Body Mass Index (BMI) were collected. Patients were divided in two different groups according to the time between injury and surgery in order to evaluate the association between the time from injury (TFI) to ACL reconstruction and the onset of lesions of the peripheral attachment of the PHMM: before and after 6 weeks, 3 months, 6 months, 1 year, and 2 years.

\section{Surgical procedure}

All surgical procedures were performed by the first Author. Patients were placed supine with a pneumatic tourniquet high on the thigh. A sand bag was positioned under the foot in order to maintain the knee at $90^{\circ}$ flexion when necessary, and a lateral support was positioned to allow valgus stress. Before ACL re- construction, a systematic diagnostic arthroscopic evaluation was performed:

- First step: a standard anterior exploration was performed through antero-medial and antero-lateral portals. The diagnosis of ACL rupture was confirmed arthroscopically. During this step, the PHMM was accurately evaluated and its stability was tested by using a probe. The presence of instability let the posterior segment of meniscus to move under the condyle by probing it [see Additional file 1].

- Second step: in all patients, we introduced the arthroscope deep in the intercondylar notch underneath the posterior cruciate ligament $(\mathrm{PCL})$ to directly visualise the $\mathrm{PM}$ compartment through an antero-lateral portal and with the knee at $90^{\circ}$ (Fig. 2). Internal tibial rotation was used to improve the visualisation of the PHMM. Later, in each patient, a No. 18 spinal needle was introduced through the PM capsule under direct arthroscopic visualisation. The spinal needle was used as a probe to better test the PHMM, its stability and its menisco-capsular junction (Fig. 3). Furthermore, as described by Strobel $^{5}$, the needle was inserted in the posterior part of the meniscal attachment, or the posterior part of the lesion, and was moved posteriorly. In the presence of a ramp lesion, this procedure opens it and, at the same time, makes its location and extention more identifiable.

- Third step: this step was performed only in patients with instability of the PHMM without any cause diagnosed. In this case, with the knee at $90^{\circ}$ flexion, we created a PM portal under direct arthroscopic visualisation with the use of a needle to localise a safe entry point (Fig. 4 a, b). We introduced the arthroscope in the PM portal to evaluate the PM compartment. Later, through intercondylar notch visualisation, we performed a debridement of the soft-tissue (Fig. 5) to identify possible hidden lesions. 


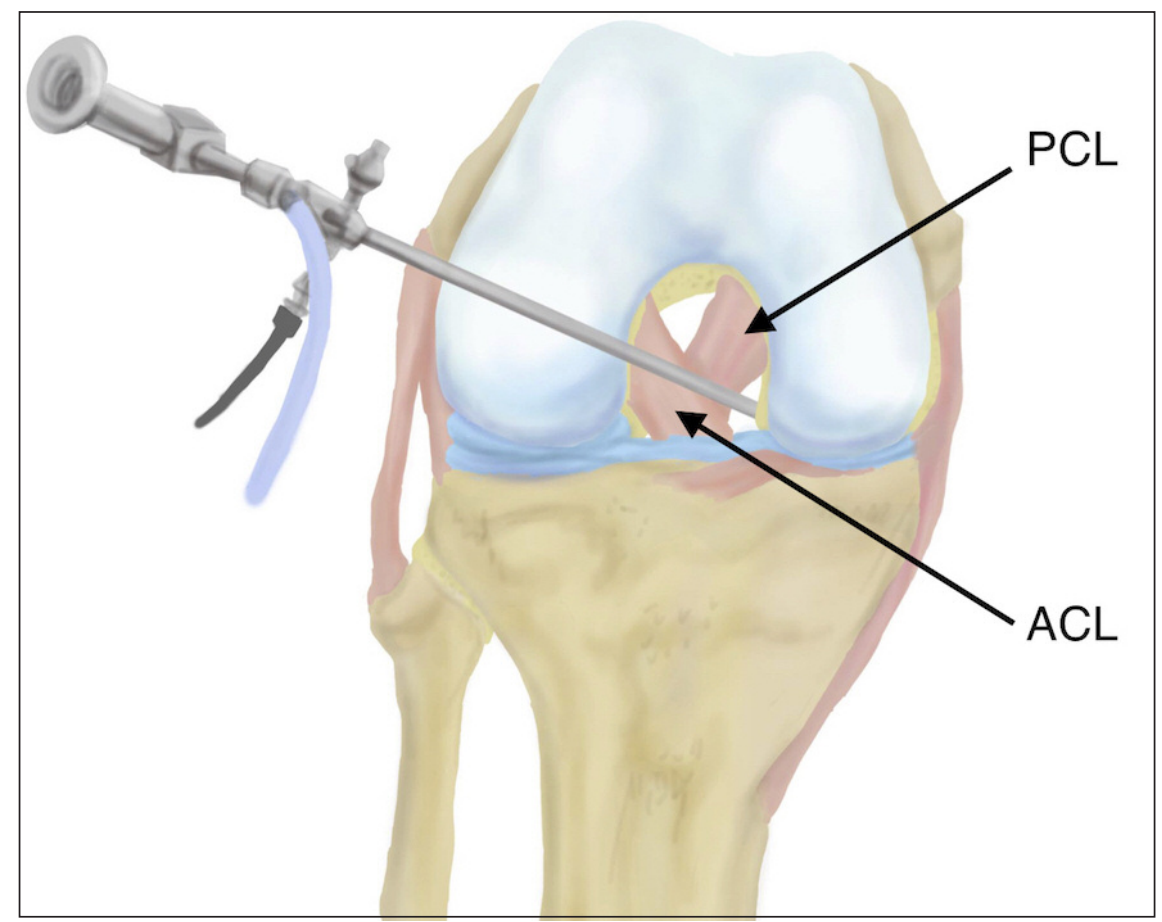

Figure 2. Arthroscope position for intercondylar view. Through an antero-lateral portal with the knee at $90^{\circ}$ flexion, the arthroscope is introduced deep in the intercondylar notch underneath the posterior cruciate ligament (PCL) in order to directly view the PM compartment.

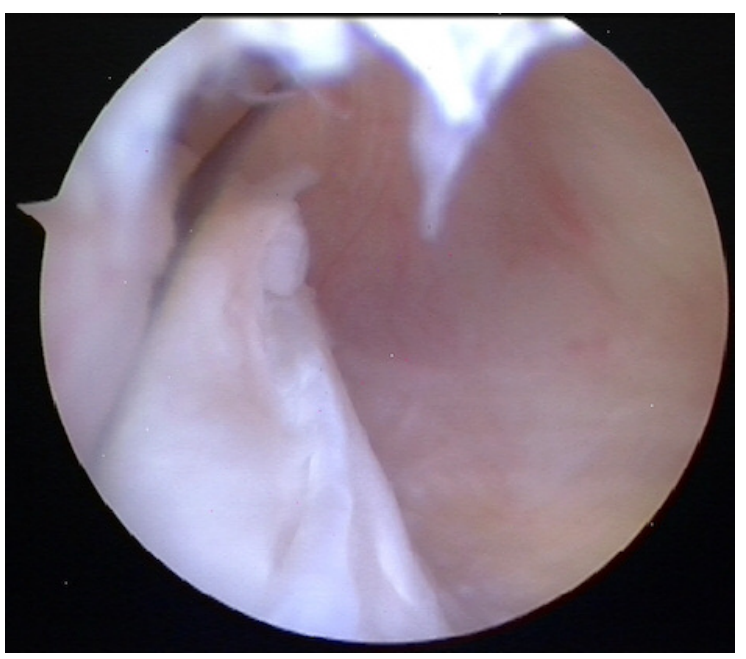

Figure 3. Use of needle as a probe. A No. 18 spinal needle is introduced through the PM capsule under arthroscopic visualisation. The spinal needle is used like a probe to better evaluate the postero-medial compartment.

For each tear of the PHMM, we reported the step in which it was diagnosed. In the presence of a ramp or hidden lesion, after freshening the lesion, we proceeded to repair it using non-absorbable suture through a PM portal by using a suture hook device. The research was ethically conduct according to international standard and journal request ${ }^{11}$. All pa- tients provided written informed consent prior to inclusion in the study. The approval from the ethics committee was waived for this study.

\section{Statistical analysis}

At descriptive statistic, quantitative variables were presented as median and interquartile range (IQR); qualitative variables were presented as number and percentage. We chose to analyse continuous variables with non-parametric tests because of the absence of a normal distribution of dataset established by Kolmogorov-Smirnov and Shapiro-Wilk tests. Statistical significance at univariate analysis was assessed by a Chi-squared or Fisher's Exact Test (when values were $<5$ ) for categorical variables, and by a Mann-Whitney $U$ test for continuous variables. $\mathrm{P}<0.05$ was considered significant. The SPSS software program v.23.0 (IBM corp. Armonk, NY, USA) was used for the database and statistics.

\section{Results}

On a total of 115 patients included, 104 (90.4\%) were males and $11(9.6 \%)$ females, with a median age of 27 (IQR: 13) and a median BMI of 24.57 (IQR: 3,47). Based on time from injury to ACL: $18(15.7 \%)$ patients were treated before 6 weeks, $36(31.30 \%)$ between 6 weeks and 3 months, $16(13.91 \%)$ between 3 months and 6 months, 15 (13.04\%) between 6 months and 1 year, 23 (20\%) between 1 year and 2 


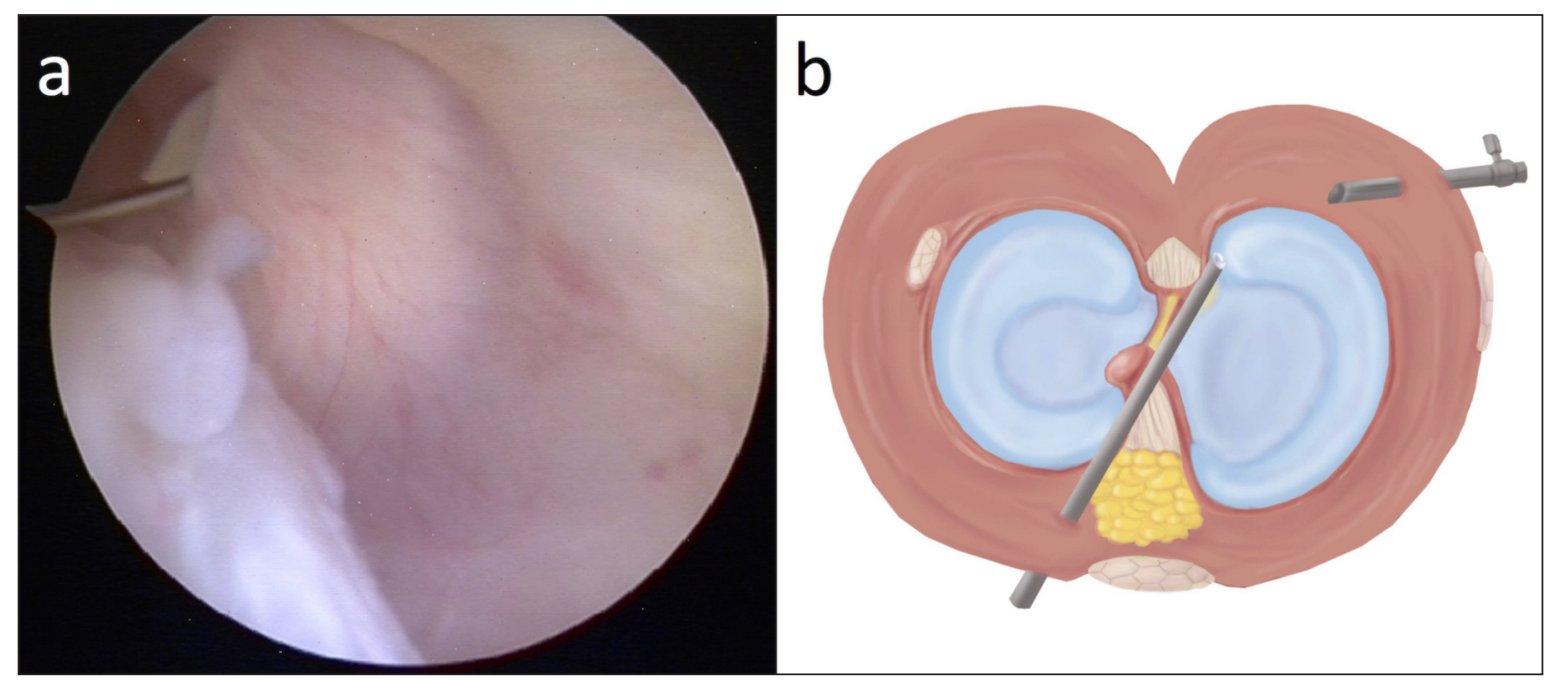

Figure 4 a, b. Postero-medial portal: entry point and its use under intercondylar visualisation. A safe entry point is localised with spinal needle under direct visualisation, then a scalpel is used to perform a postero-medial access (a); illustration of the operative use of postero-medial portal under intercondylar visualisation (b).



Figure 5. Soft tissue debridement. The soft tissue debridement of PM compartment is performed using a mechanical shaver through postero-medial portal.

years, and $7(6.1 \%)$ after 2 years. Table I shows the number and percentage of patients divided according to five different time borderlines. In our population, we recorded a $9.6 \%(11 / 115)$ prevalence of longitudinal lesions that involve the peripheral attachment of the PHMM. The diagnosis of these lesions was never made during the first step. Of the 11 lesions recorded in our population, 9 (81.82\%) were diagnosed during the second step and classified as ramp lesions, 2 $(18.18 \%$,$) were diagnosed during the third step after$ debridement of soft-tissue, and classified as hidden lesions. We have observed a greater swelling and post-operative stiffness in patients treated by using a PM access. We have not observed any complication related to possible saphenous nerve lesions in patients who were treated by using a PM portal.

Regarding the TFI, at univariate analysis only the group treated in the first 6 months revealed a statistically significant correlation $(P$-value $=0.048)$ with the presence of lesion of peripheral attachment of $\mathrm{PH}$ MM. The group of patients treated within 6 months from the injury showed a prevalence of $14.3 \%$ (10/70); the group of patients treated after 6 months, instead, showed a prevalence of $2.2 \%(1 / 45)$. No other variables showed a statistically significant correlation at univariate analysis (Tabs. II, III).

\section{Discussion}

Meniscal repair, whenever possible, must be the preferred option for patients with a meniscal lesion ${ }^{12}$. The biomechanical importance of the medial meniscus is well established. Some Authors have stressed the role of meniscocapsular ${ }^{13,14}$ and longitudinal ${ }^{15}$ tears of the PHMM on the antero-posterior knee stability in anterior cruciate ligament-deficient knees. Despite its importance, the lack of identification of $\mathrm{PH}$ $\mathrm{MM}$ lesions is the most frequent cause of mistakes in arthroscopic surgery of the knee ${ }^{16-18}$. A specific evaluation of the PHMM is difficult due to its anatomical location 6-8,19-22.

Our 3-step diagnostic and treatment algorithm is useful and effective to recognise ramp and hidden lesion. Furthermore we reported a statistically significant association between patients who underwent ACL reconstruction after 6 months from injury and a reduced prevalence of lesions of peripheral attachment of $\mathrm{PH}$ MM.

Our study has several limitations. Because of the small sample size, a separate statistical analysis of possible risk factors for ramp and hidden lesions 
Correlation between time from injury to surgery and the prevalence of ramp and hidden lesions during anterior cruciate ligament reconstruction. A new diagnostic algorithm

Table I. Categorization of study population on the basis of five different times from injury borderlines.

\begin{tabular}{lll}
\hline & \multicolumn{2}{l}{ Patients who underwent ACL-reconstruction [Number(Percentage)] } \\
\hline Time From Injury (TFI) & Before & After \\
\hline 6 weeks & $18(15.7 \%)$ & $97(84.3 \%)$ \\
3 months & $54(47 \%)$ & $61(53 \%)$ \\
6 months & $70(60.9 \%)$ & $45(39.1 \%)$ \\
1 year & $85(73.9 \%)$ & $30(26.1 \%)$ \\
2 years & $108(93.9 \%)$ & $7(6.1 \%)$ \\
\hline
\end{tabular}

Table II. Univariate analysis of possible risk factors of tears of peripheral attachment of PHMM in patients who underwent ACL-reconstruction.

\begin{tabular}{lllc}
\hline \multicolumn{3}{c}{ Patients [Number(Percentage)] } \\
\hline & $\begin{array}{l}\text { With diagnosis of lesion of the } \\
\text { peripheral attachment of PHMM }\end{array}$ & $\begin{array}{l}\text { Without diagnosis of lesion of the } \\
\text { peripheral attachment of PHMM }\end{array}$ & n.s.s. \\
\hline $\begin{array}{l}\text { Age [median (IQR)] } \\
\text { Sex[Number(Percentage)] }\end{array}$ & $27(14)$ & $27(13)$ & n.s. $^{*}$ \\
$\quad \begin{array}{l}\text { Male } \\
\text { Female }\end{array}$ & $10(9.6 \%)$ & $94(90.4 \%)$ & n.s. \\
BMI [median (IQR)] & $1(9.1 \%)$ & $10(90.9 \%)$ & $24.59(3.47)$ \\
\hline
\end{tabular}

* Fisher's exact test; n.s. - non-significant.

Table III. Univariate analysis of possible correlations between TFI and diagnosis of lesions of peripheral attachment of PHMM in patients who underwent ACL-reconstruction.

\begin{tabular}{|c|c|c|c|}
\hline & Patients [Number(Percentage)] & & \\
\hline Time from injury & $\begin{array}{l}\text { With diagnosis of lesion of the } \\
\text { peripheral attachment of PHMM }\end{array}$ & $\begin{array}{l}\text { Without diagnosis of lesion of the } \\
\text { peripheral attachment of PHMM }\end{array}$ & P-value \\
\hline $\begin{array}{l}<6 \text { weeks } \\
>6 \text { weeks }\end{array}$ & $\begin{array}{l}2(11.1 \%) \\
9(9.3 \%)\end{array}$ & $\begin{array}{l}16(88.9 \%) \\
88(90.7 \%)\end{array}$ & n.s. * \\
\hline $\begin{array}{l}<3 \text { months } \\
>3 \text { months }\end{array}$ & $\begin{array}{l}7(13 \%) \\
4(6.6 \%)\end{array}$ & $\begin{array}{l}47(87 \%) \\
57(93.4 \%)\end{array}$ & n.s. \\
\hline $\begin{array}{l}<6 \text { months } \\
>6 \text { months }\end{array}$ & $\begin{array}{l}10(14.3 \%) \\
1(2.2 \%)\end{array}$ & $\begin{array}{l}60(85.7 \%) \\
44(97.8 \%)\end{array}$ & $0.048^{*}$ \\
\hline $\begin{array}{l}<1 \text { year } \\
>1 \text { year }\end{array}$ & $\begin{array}{l}11(12.9 \%) \\
0(0 \%)\end{array}$ & $\begin{array}{l}74(87.1 \%) \\
30(100 \%)\end{array}$ & n.s. * \\
\hline $\begin{array}{l}<2 \text { years } \\
>2 \text { years }\end{array}$ & $\begin{array}{l}11(10.2 \%) \\
0(0 \%)\end{array}$ & $\begin{array}{l}97(89.8 \%) \\
7(100 \%)\end{array}$ & n.s. ${ }^{*}$ \\
\hline
\end{tabular}

* Fisher's exact test; n.s. - non-significant.

could have not been performed. Furthermore, because of the small number of lesions of the peripheral attachment of PHMM recorded, a multivariate analysis was not performed. Finally, there was a scarcity of females in our population and the absence in our study of patients with ACL tear treated conservatively was limiting because of the impossibility to diagnose a ramp or hidden lesion without arthroscopic exploration.

Bollen et al. hypothesised that PM meniscocapsular 
injury is associated with a mild anteromedial rotatory subluxation, which, if not recognised and confused with posterolateral rotatory subluxation, could lead to serious errors in surgical decision-making ${ }^{6}$.

In our practice, the systematic introduction of the intercondylar notch visualisation (second step), and the use of PM portals in patients with a posterior horn instability (third step), allowed the diagnosis of $9 \mathrm{ramp}$ lesions and 2 hidden lesions, respectively. The diagnosis of these lesions was never made during the anterior exploration of the knee (first step). Exploration of the posterior aspect of the knee must be performed routinely ${ }^{23}$, and a formal examination of the PHMM in patients who undergo $A C L$ reconstruction is always recommended ${ }^{6-8}$. According to our experience, intercondylar notch visualisation and the simultaneous probing of the PHMM using a spinal needle are appropriate to examine the meniscocapsular junction and to diagnose a ramp lesion. Regarding to hidden lesions instead, a precise diagnosis can only be carried out after a debridement of soft-tissues has been performed, making it necessary to use a PM portal. Hidden lesions should not be considered as ramp lesions covered by soft tissue (synovial or scar tissue), but as a different type of tear. Chahla et al. report that there is no actual consensus regarding the definition of meniscal ramp lesions, as different anatomical locations have been proposed as the site of injury ${ }^{24}$. Sonnery et al. have firstly hypothesised that hidden lesions represent a meniscotibial ligament injury ${ }^{8}$. We consider the disruption of the meniscotibial ligament, in some cases associated with a partial inferior longitudinal tear, a characheristic of hidden lesions; instead, a longitudinal tear of the peripheral attachment of the PHMM at the meniscocapsular junction is connected to ramp lesions (Fig. 6 a, b). Recently Thaunat et al. have proposed a classification of medial menisco-capsular tears that includes five types of lesions. In this classification, the hidden lesion is precisely described as a partial inferior lesion associated with meniscotibial ligament disruption, and high mobility at probing ${ }^{25}$. Therefore, a certain advantage of the freshening and the suture of a hidden lesion without an associated posterior horn instability is not well established by actual knowledges. According to these considerations, in our opinion, the most reliable indication for the diagnostic use of PM portals and soft-tissue debridement, in order to diagnose a possible hidden lesion that may benefit from a surgical stabilization, is an accurate evaluation of the stability of PHMM by using a probe. In our population, we recorded an interesting statistically significant correlation between patients who underwent surgery after 6 months from injury and a minor prevalence of these kinds of lesions. This result might support the hypothesis that these lesions occur during an acute trauma, at the same time of ACL injury. On the other hand, it suggests that these lesions retain a residual capacity to heal even without surgical stabilization. Because of the small sample size, it was not possible to perform a separate analysis of the influence of TFI on ramp and hidden lesions respectively. However, some indications of possible differences about the natural evolution of these two types of tears should come from the analysis of different results reported in literature. In a study of 868 patients, Liu et al. reported that the prevalence of ramp lesions increased as TFI increased 7 . Probably a possible explanation of discrepancies between our results and those of Liu et al. was suggested by Sonnery-Cottet et al. In fact, as in our study, they have also reported a higher rate of ramp and hidden lesions in the acute group $(<6$ weeks) and hypothesised that the difference with the results of the study of Liu et al. may be caused by the
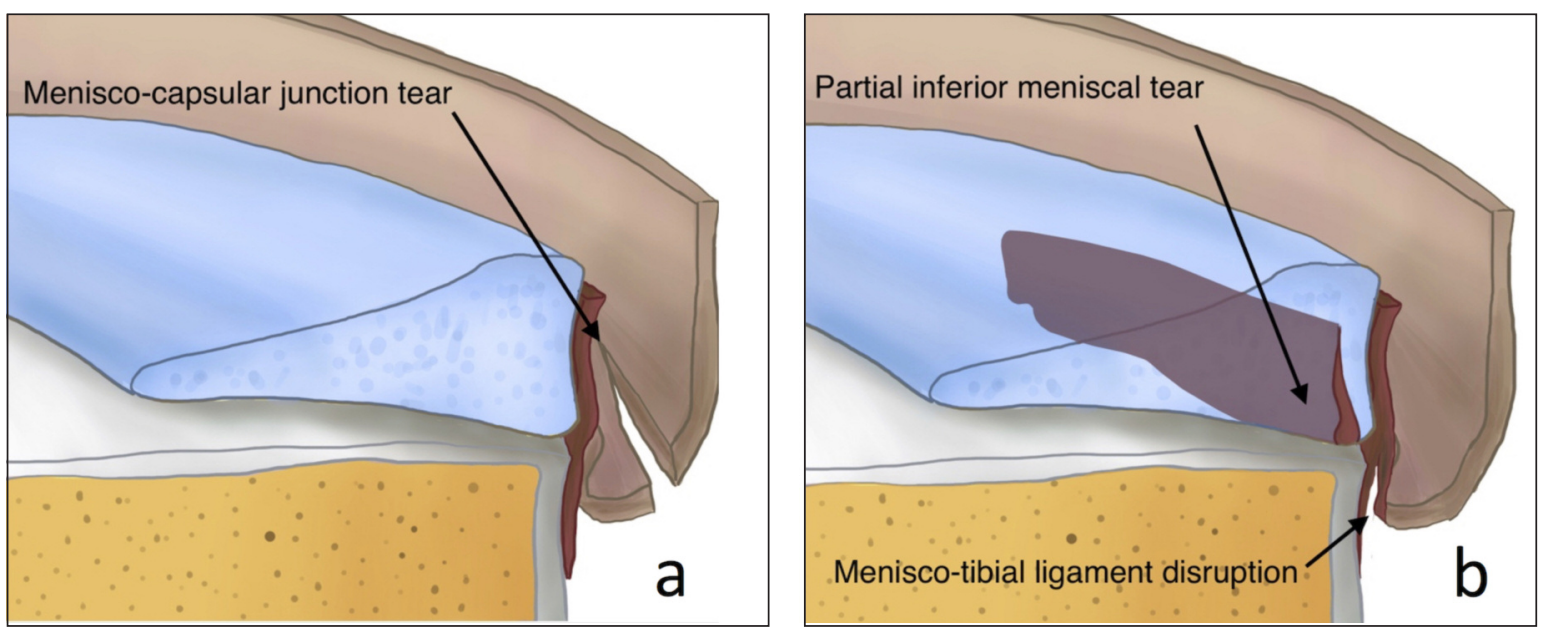

Figure 6 a, b. Possible representations of ramp and hidden lesions. A ramp lesion might represent a tear of the peripheral attachment of the PHMM at the meniscocapsular junction (a), instead a hidden lesion might be a disruption of meniscotibial ligament, in some cases associated with a partial inferior longitudinal tear of posterior horn of medial meniscus (b). The images have been recreated based on those published by Thaunat et al. Arthroscopy $2016^{25}$. 
Correlation between time from injury to surgery and the prevalence of ramp and hidden lesions during anterior cruciate ligament reconstruction. A new diagnostic algorithm

additional diagnosis of hidden lesions that were not taken into account by these Authors ${ }^{8}$. If this hypothesis was confirmed, it could be an indication that, compared to ramp lesions, hidden lesions have a greater capacity of healing without surgical stabilization, thus supporting our choice to approach ramp and hidden as different types of lesion. For sure, a huge sample is required for future researches and to standardise our diagnostic algorithm according to the time between injury and surgery; furthermore cadaveric and biomechanic studies are necessary to better understand the role and the injury mechanism of PHMM during $A C L$ rupture.

\section{Conclusions}

Ramp and hidden lesions are very common ACL rupture associated injuries. According to our diagnostic protocol, based on an accurate evaluation of PHMM by probing, a systematic use of intercondylar notch visualisation and the use of postero-medial portals and soft-tissue debridement in selected cases with a PHMM instability is a valid and safe procedure to perform during arthroscopic ACL surgical repair. A statistically significant association between patients who underwent $A C L$ reconstruction after 6 months from injury and a reduced prevalence of lesions of peripheral attachment of PHMM were reported.

\section{Conflict of interest}

The Authors declare that they have no conflict of interests.

\section{References}

1. Ahlden M, Samuelsson K, Sernert N, et al. The Swedish National Anterior Cruciate Ligament Register: A Report on Baseline Variables and Outcomes of Surgery for Almost 18,000 Patients. Am J Sports Med. 2012;40:2230-2235.

2. Granan LP, Inacio MC, Maletis GB, et al. Intraoperative findings and procedures in culturally and geographically different patient and surgeon populations: an anterior cruciate ligament reconstruction registry comparison between Norway and the USA. Acta Orthop. 2012;83:577-582.

3. Noyes FR, Chen RC, Barber-Westin SD, et al. Greater than 10-year results of red-white longitudinal meniscal repairs in patients 20 years of age or younger. Am J Sports Med. 2011;9: 1008-1017.

4. Smith 3rd JP, Barrett GR. Medial and lateral meniscal tear patterns in anterior cruciate ligament-deficient knees. A prospective analysis of 575 tears. Am J Sports Med. 2001;29:415-419.

5. Strobel M. Menisci. In: Manual of arthroscopic surgery. New York: Springer. 1988:171-178.

6. Bollen SR. Posteromedial meniscocapsular injury associated with rupture of the anterior cruciate ligament: a previously unrecognised association. J Bone Joint Surg Br. 2010;92:222223.
7. Liu X, Feng $\mathrm{H}$, Zhang $\mathrm{H}$, et al. Arthroscopic prevalence of ramp lesion in 868 patients with anterior cruciate ligament injury. Am J Sports Med. 2011;39:832-837.

8. Sonnery-Cottet B, Conteduca J, Thaunat M, et al. Hidden Lesions of the Posterior Horn of the Medial Meniscus: A Systematic Arthroscopic Exploration of the Concealed Portion of the Knee. Am J Sports Med. 2014;1-6.

9. Amin KB, Cosgarea AJ, Kaeding CC. The value of intercondylar notch visualization of the posteromedial and posterolateral compartments during knee arthroscopy. Arthroscopy. 1999;15:813-817.

10. Tolin BS, Sapega AA. Arthroscopic visual field mapping at the periphery of the medical meniscus: A comparison of different portal approaches. Arthrosc J Arthrosc Relat Surg. 1993;9:265-271.

11. Padulo J, Oliva F, Frizziero A, Maffulli N. Muscles, Ligaments and Tendons Journal - Basic principles and recommendations in clinical and field science research: 2016 update. MLTJ. 2016;6(1):1-5.

12. Vaquero J. Meniscus tear surgery and meniscus replacement. Muscles Ligaments Tendons J. Epub ahead of print. 2016. DOI: 10.11138/mltj/2016.6.1.071.

13. Stephen JM, Halewood C, Kittl C, et al. Posteromedial Meniscocapsular Lesions Increase Tibiofemoral Joint Laxity With Anterior Cruciate Ligament Deficiency, and Their Repair Reduces Laxity. Am J Sports Med. 2016;44:400-408.

14. Frizziero A, Ferrari R, Giannotti E, et al. The meniscus tear. State of the art of rehabilitation protocols related to surgical procedures. Muscles Ligaments Tendons J. 2012;2:295301.

15. Ahn JH, Bae TS, Kang K-S, et al. Longitudinal tear of the medial meniscus posterior horn in the anterior cruciate ligamentdeficient knee significantly influences anterior stability. Am J Sports Med. 2011;39:2187-2193.

16. Gillies H, Seligson D. Precision in the diagnosis of meniscal lesions: a comparison of clinical evaluation, arthrography, and arthroscopy. J bone Jt Surg Am Vol. 1979;61:343-346.

17. Gold DL, Schaner PJ, Sapega AA. The posteromedial portal in knee arthroscopy: An analysis of diagnostic and surgical utility. Arthrosc J Arthrosc Relat Surg. 1995;11:139-145.

18. Ireland J, Trickey EL, Stoker DJ. Arthroscopy and arthrography of the knee: a critical review. J bone Jt Surg Br Vol. 1980; 62-B:3-6.

19. Gillquist J, Hagberg G, Oretorp N. Arthroscopic examination of the posteromedial compartment of the knee joint. Int Orthop. 1979:3:13-18.

20. Gillquist J, Hagberg G. A new modification of the technique of arthroscopy of the knee joint. Acta Chir Scand. 1976;142:123130.

21. Mariani PP. Posterior horn instability of the medial meniscus a sign of posterior meniscotibial ligament insufficiency. Knee Surgery, Sport Traumatol Arthrosc. 2011;19:1148-1153.

22. Peltier A, Lording TD, Lustig S, et al. Posteromedial meniscal tears may be missed during anterior cruciate ligament reconstruction. Arthroscopy. 2015;31:691-698.

23. Calvisi V, Zoccali C. Arthroscopic patterns of the poster-medial aspect of the knee joint: classification of the gastrocnemiussemimembranosus gateway and its relationship with Baker's cyst. Muscles Ligaments Tendons J. 2016;6:492-498.

24. Chahla J, Dean CS, Moatshe G, et al. Meniscal Ramp Lesions: Anatomy, Incidence, Diagnosis, and Treatment. Orthop J Sport Med. 2016;4:2325967116657815.

25. Thaunat M, Fayard JM, Guimaraes TM, et al. Classification and Surgical Repair of Ramp Lesions of the Medial Meniscus. Arthrosc Tech. 2016;5:e871-e875. 